Ann. Zootech., I964, 13 (I), 5I-60

\title{
ÉTUDE DU BESOIN QUALITATIF D'AZOTE CHEZ LA TRUIE EN GESTATION ET LACTATION. COMPARAISON DE DEUX PROTÉINES : FARINE DE POISSON ET ARACHIDE
}

\author{
E. SALMON-LEGAGNEUR
}

Avec la collaboration techniqne de J. RetTagditi et IIuruette Dewulf
Station de Recherches sur l'Elevage des Porcs,
Centre national de Recherches zootechniques, Jouy-en-Josas (Seine-et-Oise)

SOMMAIRE

On a comparé sur 3 lots de truies Large IThite en reproduction et sur deux cycles complets successifs, l'effet de deux protéines, farine de poisson ct tourteau d'arachide, distribuées comme principale source d'azote au cours de la gestation ou de la lactation (aliments à 12 p. Ioo de matières azotées totales).

Par rapport au poisson, l'arachide distribuée au cours de la gestation a eu pour effet principal une très légère diminution du bénéfice corporel maternel en fin de gestation, mais n’a porté aucunement atteinte au développement et au nombre des embryons. Par contre, son utilisation au cours de la lactation a fait apparaître un déficit azoté se soldint chez la 'Yruie par une plus forte perte de poids en fin de lactation et par une certaine diminution de la production laitière au cours du $2^{\mathrm{e}}$ cycle.

Ceci permet de souligner la différence de valeur nutritionnelle qu'est susceptible de présenter une mêne protéine suivant qu'elle est utilisée au cours de la gestation ou de la lactation.

Dans nos conditions, le régime arachide pendant la gestation suivi du poisson pendant la lactation paraît le plus indiqué.

\section{INTRODUC'TION}

Depuis que Mc Col,Lum (IGI2) a montré que l'organisme pouvait utiliser pour son entretien une protéine ne convenant pas pour la croissance, comme la gélatine, les faits ne manquent pas pour prouver que la notion de valeur nutritionnelle d'une protéine peut varier suivant les circonstances. C'est ainsi qu'une protéine donnée n'aura pas la même valeur biologique suivant l'espèce qui l'utilise, l'âge des sujets et la fonction envisagée. Inversement, ceci peut servir à montrer que les besoins qualitatifs d'azote d'un animal sont eux-mêmes très variables.

Or, dans ce domaine si les besoins du jeune Porc en croissance sont assez bien 
connus, on ne dispose que de peu d'informations pour le Porc plus âgé et encore moins pour la Truie en reproduction.

Dans un travail antérieur (SALMON-LEGAGNEUR, I96I $b$ ), confirmé par RomBAU'SS (Ig62), nous avions montré que la gestation s'accompagnait chez la Truie d'une réduction du besoin quantitatif d'entretien et l'on peut se demander si cette épargne se poursuit sur le plan qualitatif. Autrement dit, la Truie est-elle capable de tirer parti de protéines habituellement considérées conme déséquilibrées, ou, ce qui revient au même, la valeur nutritionnelle de telles protéines est-elle la même chez la 'Truie gestante ou en lactation, que chez l'animal en croissance ou au repos.

Dans le but de préciser ce point, nous avons testé chez des truies en reproduction deux protéines connues pour la différence de leur valeur biologique chez le Rat et chez le Porc en croissance : le tourteau d'arachide et la farine de poisson.

Parmi de nombreuses études, en effet, on peut retenir celles de Jones et al., (I962), Combs (I962) et Rírát (I963) qui toutes ont montré que l'arachide provoquait des vitesses de croissance et des efficacités alimentaires très inférieures à celle du poisson chez des pores de différents âges, pesant de 3 à $60 \mathrm{~kg}$.

Ces mêmes protéines ont été utilisées dans notre expérience comme principales sources azotées de régimes bien équilibrés par ailleurs. Leurs valeurs respectives ont été étudiées sur des truies au cours de deux cycles de reproduction successifs, gestation et lactation.

Les critères retenus comme témoins de l'efficacité de ces aliments étaient les variations de poids et d'adiposité aux différents stades physiologiques, les résultats de parturition et les productions laitières.

\section{MATÉRIEL, E'T TECHNIQUES}

\section{A) Animaux}

46 truies de race $\operatorname{Larg}_{0}$ White issues de notre troupeau expérimental ct n'ayant pas encore reproduit ont été utilisées dans cette expérience. Ces truies étaicnt élcvées d'une façon identique et recevaient une même ration équilibrée jusqu'à l'accouplement qui était pratiqué au deuxième œstrus après la puberté (poids moyen des animaux : $\mathrm{I} 35 \mathrm{~kg}$ ).

Les animaux étaient alors répartis en 3 lots expérimentaux de I 2 truies recevant, soit un régime ì base d'arachide, soit un régime à base de farine de poisson en suivant les 3 combinaisons suivantes :

Lot A-P': Arachide pendant la gestation, poisson pendant la lactation.

Lot P-P : Poisson pendant la gestation et la lactation.

Lot P-A : Poisson pendant la gestation, arachide pendant la lactation.

En outre, deux lots témoins, de même poids initial, de 5 truies non gestantes étaient constitués et recevaient pendant la durée d'une gestation, soit I 15 jours, le régime arachide ou le régime poisson. Sauf pour ces deux derniers lots, et après une période de repos de la durée d'un ostrus après le sevrage, l'expérience était répétée au cours d'un deuxième cycle de reproduction avec les mêmes animaux et les mêmes régimes. Toutefois, deux animaux par lot durent alors être éliminés pour raisons diverses (défauts de reproduction).

\section{B) Aliments}

Les animaux étaient nourris individuellement et recevaient, quelle que soit leur affectation, des quantités journalières d'aliment constantes : $3 \mathrm{~kg}$ pendant la getsation et $4,5 \mathrm{~kg}$ pendant la lactation. Cette dernière ration, légèrement inférieure peut-être aux rations habituellement distribuées aux animaux de ce poids, correspondait au maximum d'ingestion consenti par les truies recevant l'aliment à l'arachide. 
Dans tous les cas, les rations étaient exactement mesurées et distribuées en deux repas par jour, sous forme d'une pâtée épaisse $(\mathrm{I} / 3$ farine $+2 / 3$ eau) préparée au moment de l'emploi. Il était tenu compte des " refus " éventuels qui étaient déduits dans le calcul des ingérés.

La composition des deux régimes utilisés est indiquée au tableau I.

TABLEAU I

Composition des régimes (secs)

\begin{tabular}{|c|c|c|}
\hline & Arachide & Poisson \\
\hline \multicolumn{3}{|l|}{ Aliments } \\
\hline Manioc ................ & 72 & 76 \\
\hline 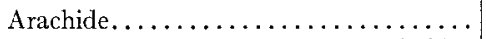 & 16 & - \\
\hline Farine de poisson du Pérou (anchois). & - & 12 \\
\hline 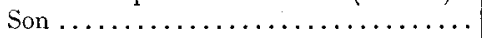 & 7 & 7 \\
\hline Mélange minéral complet .......... & 3 & 3 \\
\hline \multirow{2}{*}{ Complément vitaminique $\ldots \ldots \ldots \ldots \ldots$} & 2 & 2 \\
\hline & 100 & 100 \\
\hline \multicolumn{3}{|l|}{ Composition chimique } \\
\hline Eau $\ldots \ldots \ldots \ldots \ldots \ldots \ldots \ldots$ & 11,7 & 11,7 \\
\hline Matière azotée totale $(\mathrm{N} \times 6,25) \ldots \ldots$ & 11,7 & 11,8 \\
\hline Cellulose .................... & 4,1 & 3,1 \\
\hline Matière grasse $\ldots \ldots \ldots \ldots \ldots \ldots \ldots \ldots$ & 1,8 & 2,1 \\
\hline Minéraux $\ldots \ldots \ldots \ldots \ldots \ldots \ldots \ldots$ & 5,8 & 6,1 \\
\hline \multirow[t]{3}{*}{ Extractif non azoté.............. } & 64,6 & 65,2 \\
\hline & 100 & 100 \\
\hline & & \\
\hline
\end{tabular}

C) Mesures effectuées

Pendant toute la durée de l'expérience, chaque truie était pesée une fois par semaine à heure fixe. En outre, à la parturition les truies étaient pesées immédiatement après l'expulsion du placenta. Les porcelets étaient alors dénombrés et pesés. Pendant la lactation qui durait 8 semaines, le nombre de porcelets de chaque portée était, dans la mesure du possible, égalisé après 48 heures à 9 porcelets au cours de la première lactation et à ıo au cours de la seconde. Cette mesure était destinée à éviter l'influence du nombre de porcelets sur la production laitière des mères.

Le poids moyen des porcelets aux différents âges était noté et servait de base pour l'estimation des productions laitières (SALMON-LEgAGNEUR, 1958). En outre, un échantillon hebdomadaire de lait était prélevé sur chaque truie, au cours de la ire lactation seulement, et analysé pour sa teneur en azote et en lipides.

Les variations de l'adiposité des truies étaient suivies par mesure aux ultrasons de l'épaissenr du tissu sous-cutané dorso-lombaire. Cette mesure était effectuée au début, au milieu et à la fin de chaque période.

\section{RÉSULTATS}

\section{A) Variation de poids des truies}

Le tableau 2 et la figure I rapportent l'évolution du poids des truies de chaque lot au cours des différentes périodes de chacun des deux cycles considérés.

Le résultat le plus remarquable concerne la différence de comportement entre les témoins et les gestantes (premier cycle). Quel que soit le régime, les truies ges- 
tantes ont effectué des gains de poids très supérieurs à celui des témoins, ce qui montre bien que l'efficacité d'une même protéine est plus grande pendant la gestation que pendant la période de repos sexuel.

TABLEAU 2

Variation de poids des truies

\begin{tabular}{|c|c|c|c|c|c|c|c|c|}
\hline \multirow[b]{2}{*}{ Lots } & \multicolumn{4}{|c|}{ Poids (kg) } & \multicolumn{4}{|c|}{ Variations $(\mathrm{kg})$} \\
\hline & $\begin{array}{l}\text { Accou- } \\
\text { plement }\end{array}$ & $\begin{array}{c}\text { Avant } \\
\text { parturition }\end{array}$ & $\begin{array}{c}\text { Après } \\
\text { parturition }\end{array}$ & Sevrage & $\begin{array}{l}\text { Gain } \\
\text { total } \\
\text { gestation }\end{array}$ & $\mid \begin{array}{c}\text { Gain } \\
\text { net } \\
\text { gestation }\left({ }^{1}\right)\end{array}$ & $\begin{array}{c}\text { Perte } \\
\text { lactation }\end{array}$ & Bilan \\
\hline Témoin $\mathrm{P}$ & 130,0 & 178,2 & - & - & 48,2 & - & - & - \\
\hline Témoin A & 130,0 & 180,0 & - & - & 50,8 & - & - & - \\
\hline \multicolumn{9}{|l|}{$1^{\text {er }}$ cycle } \\
\hline A-P & 135,5 & 210,4 & 191,4 & 171,6 & 74,9 & $55,9(*)$ & 19,8 & 36,1 \\
\hline $\mathrm{P}-\mathrm{P}$ & 134,8 & 213,7 & 196,9 & 176,0 & 78,9 & 62,1 & 20,9 & 41,1 \\
\hline P-A & 132,9 & 213,1 & 197,2 & 147,2 & 80,2 & 64,3 & $50,0(* *)$ & $14,3\left(^{*}\right)$ \\
\hline \multicolumn{9}{|l|}{$2^{2}$ cycle } \\
\hline A-P & 185,9 & 264,3 & 244,5 & 220,7 & $78,4(*)$ & $58,6 \quad(*)$ & 23,8 & $3 \prime, 8$ \\
\hline $\mathrm{P} \cdot \mathrm{P}$ & 192,9 & 280,2 & 261,7 & 222,7 & 87,3 & 68,8 & 39,0 & 29,8 \\
\hline P-A & 177,5 & 268,0 & 249,7 & 184,0 & 90,9 & 72,0 & $70,5(* *)$ & $2,5\left(^{*}\right)$ \\
\hline
\end{tabular}

(*) Différence significative.

(**) Différence très significative.

(1) Poids après parturition - poids à l'accouplement.

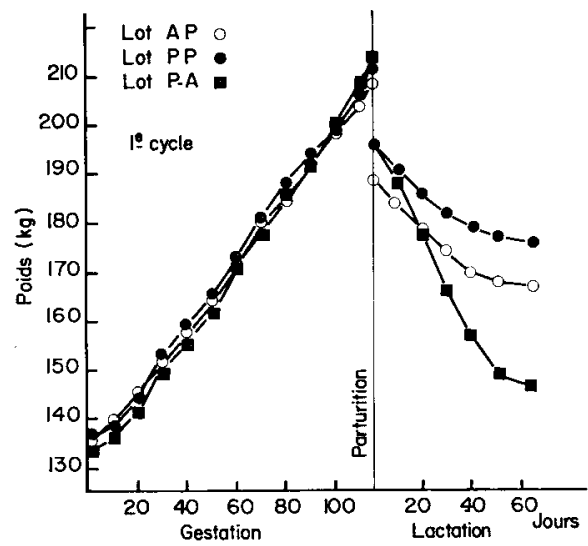

FIG. I. - Evolution du poids vif des truies au cours du premier cycle

Par ailleurs, la comparaison des deux protéines entre elles fait ressortir les points suivants : aucune différence en ce qui concerne les témoins et légère influence en faveur du poisson chez les gestantes. Cette différence tend à s'accuser au cours du 
deuxième cycle de reproduction (elle passe de 5 p. Ioo à I 2 p. Ioo du gain total) et elle est plus sensible pour le gain net maternel, ou anabolisme de gestation, que pour le gain total, qui englobe les productions fotales.

Mais c'est surtout au cours de la lactation que les différences sont les plus importantes : la perte de poids est considérablement plus élevée chez les animaux recevant de l'arachide pendant la lactation, que chez ceux qui reçoivent du poisson, quel que soit le régime antérieur de ces derniers. La différence est de l'ordre de Ioo p. Ioo et paraît également plus élevée au cours du deuxième cycle que du premier.

Il en résulte un bilan final, gain entre l'accouplement et le sevrage, plus élevé chez tous les animaux ayant reçu du poisson pendant la lactation.

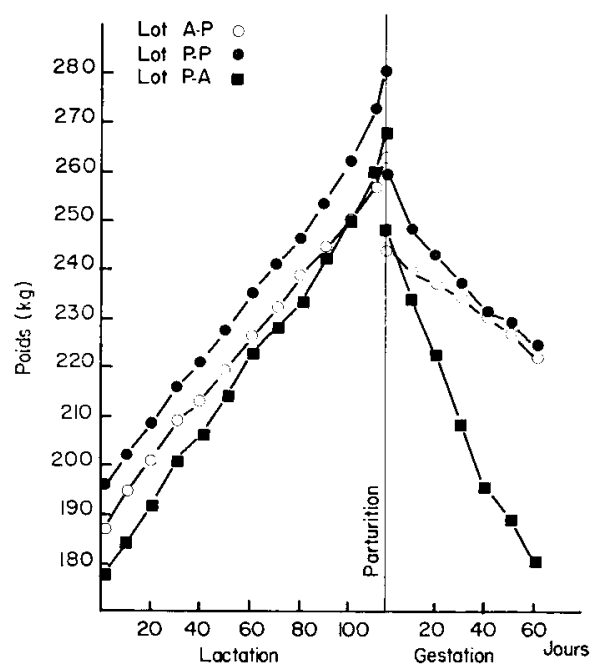

Fic. 2. - Erolution du poids vif des truies au cours du deuxième cycle

\section{B) Efficacités protidiques}

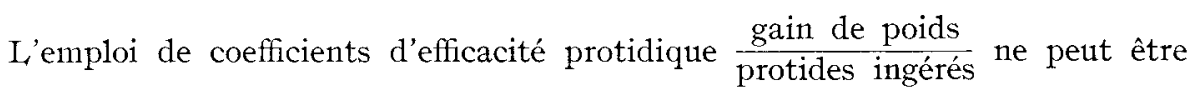
fait qu'avec réserve. Nous n'ignorons pas, dans le cas présent, tout ce qu'il comporte comme inexactitude et insuffisance car, d'une part il est maintenant connu que les variations de poids au cours de la reproduction de la Truie n'affectent pas dans les mêmes proportions les différents tissus et que ceux-ci n'ont pas une composition constante (SALMON-LEGAGNEUR, Ig6I b) ; par ailleurs, dans le cas de lactation, le calcul de tient pas compte de l'azote exporté dans le lait.

Toutefois, il s'agit d'un procédé commode pour comparer la valeur nutritionnelle des régimes et, à titre indicatif, nous rapportons au tableau 3 , les coefficients d'efficacité protidique aux différents stades.

Ces valeurs soulignent tout d'abord la meilleure utilisation des protéines par les truies gestantes par rapport aux truies au repos et montrent, en outre, que les différences entre protéines varient suivant la période considérée : mêmes valeurs 
au repos, légère différence pendant la gestation et supériorité marquée des protéines de poisson pendant la lactation (pendant la lactation les C.E.P. sont négatifs, car il s'agit de perte de poids, et d'autant plus élevés que la protéine est moins efficace).

TABLEAU 3

Coefficients d'efficacité protidique

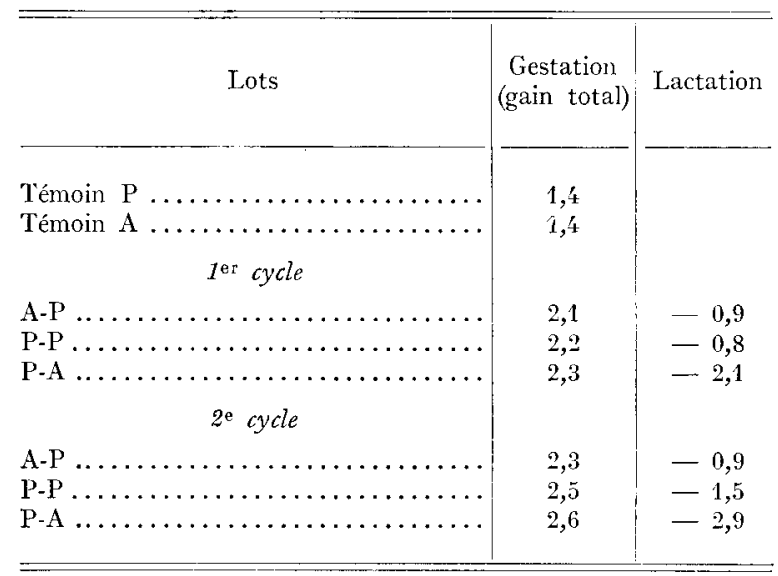

TABLEAU 4

Variation des épaisseurs du tissu adipeux dorsal

\begin{tabular}{|c|c|c|c|c|c|c|c|c|c|}
\hline \multirow{3}{*}{ Lots } & \multicolumn{3}{|c|}{ Épaisseurs (mm) } & \multicolumn{6}{|c|}{ Variation en $\mathrm{mm}$ et en p. 100 de l'épaisseur initiale } \\
\hline & $\begin{array}{l}\text { Accou- } \\
\text { plement }\end{array}$ & $\begin{array}{c}\text { Partu- } \\
\text { rition }\end{array}$ & Sevrage & \multicolumn{2}{|c|}{ Gain gestation } & \multicolumn{2}{|c|}{ Perte lactation } & \multicolumn{2}{|c|}{ Bilan } \\
\hline & & & & $(\mathrm{mm})$ & (p. 100) & $(\mathrm{mm})$ & (p. 100) & $(\mathrm{mm})$ & (p. 100) \\
\hline Témoin P.. & 36,0 & $4,5,0$ & - & 9,0 & $25(*)$ & - & - & - & - \\
\hline $\begin{array}{c}\text { Témoin A .. } \\
1^{\mathrm{er}} \text { cycle }\end{array}$ & 34,5 & $\begin{array}{l}42,3 \\
1\end{array}$ & - & 7,8 & $23(*)$ & - & $\rightarrow$ & $\longrightarrow$ & 一 \\
\hline$A-P \ldots \ldots \ldots$ & 37,8 & 42,3 & 37,0 & 4,5 & $12(*)$ & 5,3 & 14 & $-0,8$ & -2 \\
\hline P-P ....... & 37,3 & 43,6 & 39,0 & 6,3 & 17 & 4,6 & 13 & $+1,7$ & +4 \\
\hline $\begin{array}{c}\mathrm{P}-\mathrm{A} \ldots \ldots \\
2^{\mathrm{e}} \text { cycle }\end{array}$ & 36,1 & 42,6 & 34,9 & 6,5 & 17 & 7,7 & $20\left(^{*}\right)$ & $-1,2$ & -3 \\
\hline A-P....... & 40,9 & 46,5 & 40,5 & 5,6 & 13 & 6,0 & 14 & $-0,5$ & -1 \\
\hline P-P ...... & 43,3 & 50,2 & 43,1 & 6,9 & 16 & 7,1 & & $-0,2$ & -1 \\
\hline P-A........ & 39,1 & 46,9 & 37,9 & 7,8 & 20 & 9,0 & $23\left(^{*}\right)$ & $-1,2$ & -3 \\
\hline
\end{tabular}

(1) Différence significative. 


\section{C) Compositions corporelles}

Les variations de l'épaisseur du tissu adipeux sous-cutané dorsal renseignent assez bien sur l'évolution de l'adiposité totale et donc, indirectement, sur la composition corporelle globale. Le tableau 4 rapporte les variations observées.

I)'une manière générale, les différences entre lots sont peu importantes et peu significatives. On peut noter toutefois que le gain de tissu adipeux parait plus important chez les témoins que les gestantes et, chez ces dernières, plus élevé avec le régime poisson. Pendant la lactation, au contraire, le régime arachide parait augmenter la mobilisation des lipides (fig. 3).

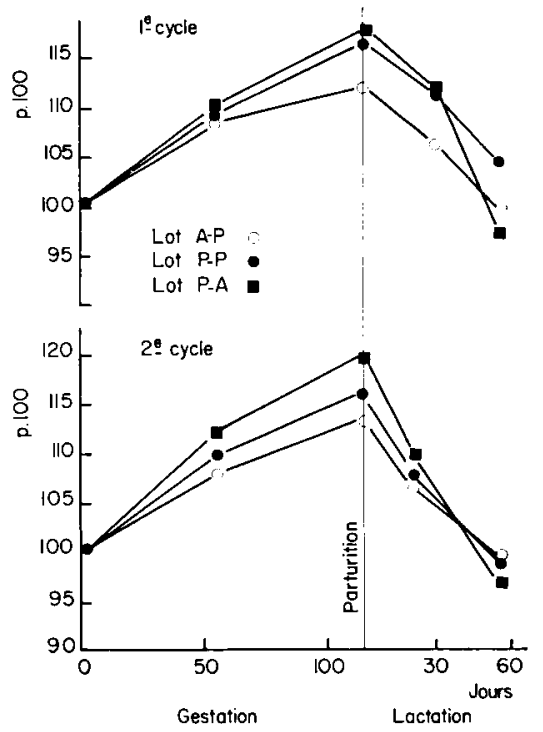

Fig. 3. - Évolution de l'adiposité (p. Ioo de l'épaisseur initiale du tissu adipeux sous-cutané dorsal)

\section{D) Parturition}

Les principales caractéristiques des portées à la naissance sont rapportées au tableau 5 .

Mise à part l'augmentation générale du poids des productions au cours du deuxième cycle, qui semble résulter d'une plus grande prolificité, on n'observe aucune différence significative entre les différents lots.

On remarquera en particulier que les porcelets du lot nourri à l'arachide pendant la gestation sont aussi nombreux, et même légèrement plus lourds, que ceux des lots nourris au poisson. 
TABLEAU 5

Produits de la conception

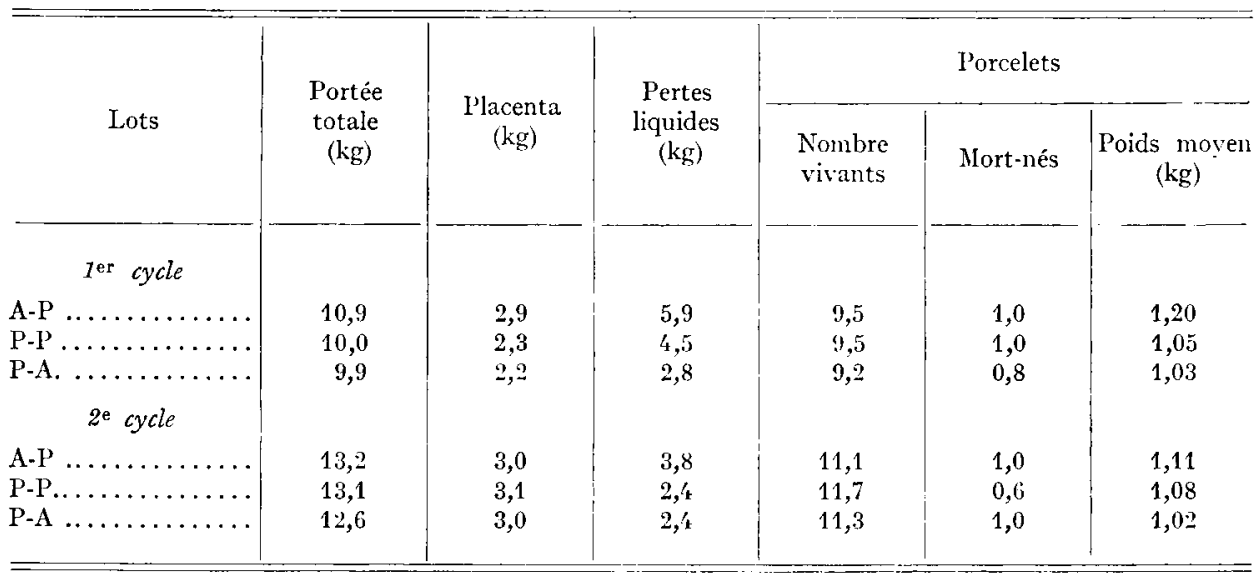

\section{E) Lactation}

Comme le montre le tableau 6 , on ne constate aucune différence dans la croissance des porcelets et dans la production laitière quantitative et qualitative des truies au cours du premier cycle de reproduction.

Il faut attendre le deuxième cycle pour voir apparaître une légère diminution de la quantité de lait produite dans le lot recevant de l'arachide pendant la lactation.

TABI.EAU 6

Productions laitières

\begin{tabular}{|c|c|c|c|c|c|c|}
\hline \multirow[b]{2}{*}{ Lots } & \multicolumn{2}{|c|}{ Croissance des porcelets } & \multirow{2}{*}{$\begin{array}{c}\text { Nombre de } \\
\text { porcelets } \\
\text { sevrés }\end{array}$} & & uction la & \\
\hline & $\begin{array}{l}\text { Poids moyen } \\
\text { à } 21 \mathrm{j}(\mathrm{kg})\end{array}$ & $\begin{array}{l}\text { Poids moyen } \\
\text { au sevrage }(\mathrm{kg})\end{array}$ & & $\begin{array}{l}\mathrm{Kg} \\
\text { lait }\end{array}$ & $\begin{array}{l}\text { Matière } \\
\text { azotéc } \\
\text { p. } 100\end{array}$ & $\begin{array}{c}\text { Matière } \\
\text { grasse } \\
\text { p. } 100\end{array}$ \\
\hline $7^{\mathrm{er}}$ cycle & & & & & & \\
\hline$A-P \ldots \ldots \ldots$ & 4,7 & 15,7 & 8,0 & 305 & 5,6 & 7,0 \\
\hline P-P $\ldots \ldots \ldots$ & 4,5 & 15,1 & 7,7 & 287 & 5,6 & 6,0 \\
\hline $\begin{array}{c}\mathrm{P}-\mathrm{A} \ldots \ldots \ldots \\
2 \mathrm{cycle}\end{array}$ & 4,7 & $15,{ }^{\prime}$ & 7,7 & 295 & 5,5 & 6,8 \\
\hline$A-P \ldots \ldots \ldots$ & 4,8 & $17, \bar{j}$ & 9,0 & 311 & - & - \\
\hline P-P $\ldots \ldots \ldots$ & 5,3 & 18,8 & 8,8 & 359 & - & - \\
\hline P-A $\ldots \ldots \ldots$ & 4,6 & 17,7 & 8,2 & 315 & 一 & 一 \\
\hline
\end{tabular}




\section{DISCUSSION}

Comme dans nos expériences précédentes (SALMON-LEGAGNEuR, Ig6I $a$, Ig63), la principale influence d'une modification du régime alimentaire de gestation, ou de lactation, réside dans les variations, gains ou pertes, de poids corporel des mères.

Dans les limites de nos expériences, tout semble se passer comme si l'accumulation ou la mobilisation des tissus de réserve, dont nous avons déjà souligné la grande plasticité chez la Truie (SAlmon-LEGAGNEUR, I960), se fait dans la limite des disponibilités laissées par les deux fonctions prioritaires que sont, chez la femelle en reproduction, la croissance des embryons et la production laitière.

Cette hypothèse permet de comprendre, comme l'avait déjà souligné PIkE (1954) chez la Ratte, que toute amélioration du régime alimentaire se traduit d'abord par une augmentation des réserves anabolisées pendant la gestation et une diminution du catabolisme de lactation.

C'est dans cette mesure, que la présente expérience met en relief la variabilité de la notion de valeur nutritionnelle d'une protéine en fonction de l'état physiologique de l'animal. On constate en effet que deux protéines dont la différence des valeurs biologiques est bien connue chez le jeune animal en croissance, mais qui présentent un égal pouvoir de stimulation chez l'animal plus âgé (témoins de $130 \mathrm{~kg}$ ) peuvent également se comporter différemment suivant qu'elles sont offertes pendant la gestation ou la lactation.

Pendant la gestation, le seul effet des protéines de poisson par rapport à celles de l'arachide est une très très légère augmentation de l'anabolisme gravidique maternel, à la fois azoté et lipidique, alors que les produits de la conception, et notamment le poids des foetus, ne sont absolument pas modifiés. Des constatations analogues avaient déjà été faites par FowlEk (I954) et par GARD et al., (I955). Ce dernier notamment avait montré que chez la Truie l'addition de 3 p. Ioo de "fish soluble" à un régime ne comportant que des protéines d'origine végétale n'améliorait en aucune façon le nombre et le poids des embryons.

Chez les autres espèces, KLostermax et al., (I95I) arrivent à une conclusion semblable arec la Brebis, mais il faut surtout citer Champigry et Jacouot (I96I) qui ont trouvé que le principal effet d'une limitation quantitative et qualitative de l'ingéré azoté sous forme de gluten, en comparaison avec de la caséine, chez la Ratte gravide, résidait dans la perte de poids relative des mères, alors que les produits de la conception conservaient un développement parfaitement normal.

Il apparaît en définitive que, quelle que soit l'espèce, la différence d'efficacité pour le maintien de la gestation, de deux protéines d'origine et de valeur très différentes, comme l'arachide et le poisson, est peu importante puisqu'elle n'est ressentie, faiblement, qu'au niveau de l'anabolisme maternel.

Il en va tout autrement au cours de la lactation. Si en effet, au cours du premier cycle de reproduction, il n'apparaît aucune différence dans les productions laitières des truies recevant les différents régimes, ce résultat n'a pu être acquis qu'au prix d'une mobilisation beaucoup plus élevée des tissus corporels chez les truies nourries à l'arachicle. Ce résultat reflète donc bien une nette différence de valeur biologique pour la production laitière entre les protéines utilisées, mais celle-ci a été en partie 
masquée par le rôle de tampon que jouent les réserves tissulaires. Ceci n'est pas nouveau (GÜTTE I957) et met l'accent sur la participation, habituelle ou non, de l'azote corporel à la synthèse des protéines du lait et de l'état dynamique, immédiatement disponible, des réserves azotées édifiées au cours de la gestation.

Cette mobilisation des tissus, pour pallier l'insuffisance qualitative du régime azoté s'accompagne, chez la 'Truie, d'une certaine fonte lipidique, plus élevée dans le cas d'une protéine de mauvaise qualité. Ceci confirme la liaison qui existe entre les métabolismes azoté et énergétique. Il faut remarquer toutefois qu'en valeur relative, rapportée à la perte de poids totale, les truies ont perdu deux fois moins de tissu adipeux avec le régime à l'arachide qu'avec le poisson. Ceci indique donc que la relation est complexe.

Au cours du deuxième cycle de reproduction, les phénomènes sont encore accentués et l'on note, à côté de la perte de poids plus élevée des animaux du lot P-A, une certaine diminution de la production laitière due en partie au nombre plus faible de porcelets sevrés. Peut-être faut-il voir ici la conséquence d'effets cumulatifs avec le temps? Plus simplement, on peut imaginer que les animaux plus lourds qu'au premier cycle, mais recevant la même ration, ont eu des déficits plus importants dus à une augmentation du besoin d'entretien.

Quelles que soient les circonstances, on constate donc que chez la Truie en lactation des protéines de valeurs diverses peuvent avoir des effets différents. Ceci confirme les observations de WERNER et HENNIG (I960), ou celles faites par MUEI,LER et Cox (I937) sur la Ratte avec des régimes à base de levure et rejoint également les opinions émises par différents auteurs quant à la valeur biologique des protéines au cours de la lactation (MAynard, I962, BREIREM, I957).

On ne peut manquer de souligner ici les différences qui apparaissent en fonction de l'état physiologique. C'est ainsi que, dans notre expérience, le choix des protéines, qui n'a eu aucune influence lorsqu'il s'agissait d'animaux en fin de croissance ou à l'entretien, a marqué très faiblement les résultats de la gestation et d'une façon beaucoup plus nette ceux de la lactation. Cette constatation, qui reflète la variabilité des valeurs biologiques, pərmat d'établir une certaine hiérarchie des besoins qualitatifs suivant les fonctions considéréss. Pour la truie en reproduction, les besoins iraient croissants de l'entretien à la production laitière.

Si ces conclusions se trouvaient confirmées, certaines conséquences pourraient en découler pour l'alimentation des animaux. C'est ainsi qu'on pourrait envisager, au cours de la gestation de la Truie, l'emploi de protéines végétales seules, moins coûteuses, mais tout aussi efficaces que les protéines d'origine animale. Par contre, au cours de la lactation, davantage d'attention devrait être apporté au choix et à l'équilibre des protéines de la ration.

Reçu pour publication en décembre 1963 .

\section{SUMMARY}

QUALITATIVE NITROGEN REQUIREMENTS IN PREGNANT AND LACTATING SOWS. A COMPARISON BETWEEN TWO PROTEINS : GROUND NUT AND FISII MEAL

This experiment was carried out to compare the influence of a high quality protein (fish meal) to a low quality protein (extracted ground nut meal) during pregnancy and lactation in the Sow. 
46 Large White sows were alloted in 3 experimental and 2 control groups receiving during 2 reproductive cycles one of the II $p$. Ioo protein diets as follows :

Group A-P : Gestation : peanut meal, lactation : fish meal.

Group P-P : Gestation : fish meal, lactation : fish meal.

Group P-A : Gestation : fish meal, lactation : peanut meal.

Control P : Fish meal during II4 days.

Control $A$ : Peanut during iI4 days.

Controls exhibited the same live weight gain. In the experimental groups it appears that fish meal during gestation slightly increases total gain of the sows at parturition, but did not improve either weight or number of piglets born.

On the other hand, when peanut meal was given during lactation a sharp loss of live weight for the two cycles and a decrease of milk production in the $2^{\circ}$ cycle occurred.

From these results, it was concluded that the two proteins have nearly the same nutritive value for maintenance and pregnancy, but were different for lactation.

In our conditions, peanut during pregnancy followed by fish meal during lactation, was thought the most advantageous diet.

\section{RÉFÉRENCES BIBLIOGRAPHIQUES}

BREIREM K., 1957. Nutrition and lactation in domestic animals and particularly in the cow. Ann. Nutr. Alim., 11, 3-32.

Champigny O., Jacquot R., ig6r. Comportement remarquable de la Ratte gestante en déficience quantitative et qualitative de protides alimentaires. C. R. Acad. Sci. Paris, 252, 2765-2567.

Combs G. E., Waldace H. D., I962. Peanut meal as a source of protein in pigs starter and growth ration. Canad. Anim. Sci, 21, 95-97.

Fowler S. H., ROBERTSON G. L., 1954. Some effects of source protein and an antibiotic on reproductive performances in gilts. Canad. Anim. Sci., 13, $949-954$.

Gard D. I., Terrill S. W., Becker D. E., I955. Effects of the addition of dehydrated alfalfa meal, fish solubles and vitamine $B_{13}$ to a purified diet for sows. Canad. Anim. Sci., 14, 562-572.

Gutte J. O., Lenkeit W., 1957. Die Verwertung des Proteins zur Milcheiweissynthèse. Z. Tiérern., $12,52-57$.

Jones A. S., Hepburn W. R., Gadenhead A., Boyne A. W., I962. The effect of variation of protein quality and protein level in diet on the performances of young pigs. Anim. Prod., 4, I85-I93.

Klosterman E. W., Buchanan M. L., Bolen D. W., I95I. Levels and sources of protein in rations for pregnant ewes. Canad. Anim. Sci., 10, г63-17o.

Maynard L. A., Loosli J. K., 1962. Animal mutrition. 5th ed. Mc Graw Hill book, N. Y, 533 pp.

Mc Collum F. V., Steenbock H., igi2. Cité par Mrtchell H. H. in Albanese A., Protein and amino acids nutrition, Academic Press, N. Y.

Mueleter A. J., Cox W. M., 1937. The effect of changes in diet on the volume and composition of rat milk. J. Biol. Chem., 117, LXXII.

PIKE R. L., SUder H. B., Ross M. L., I954. The influence of protein and energy intakes upon nitrogen retention in pregnant rats. J. Nutr., 52, 297-309.

RÉRAT A., I963. Rapport particulier sur les possibilités d'accroitre l'efficacité de l'utilisation des aliments. Première Conf. mondiale de Zootechnie, Rome, vol. II, 89-105.

Rombauts P., ig62. Évolution de l'anabolisme gravidique chez la Truie en fonction de l'âge de l'animal. Ann. Zootech., 11, 39-5r.

Salmon-Legagnetr E., i958. Observations sur la production laitière des truies. Ann. Zoolech., 7, I 43-162.

SALMON-LEGagneUr E., Gomez G., JaCQuot R., i960. Influence de la suralimentation en fin de gestation sur la production laitière de la Truie. C. R. Aacd. Agric. France, 46, 445-45r.

SaLmon-LegagNeUR E., Jacedot R., I96I $a$. Quelques aspects des relations nutritionnelles entre la gestation et la lactation chez la Truie. C. R. Acad. Sci. Paris, 253, 1618-1620.

SALMON-LEgagneli E., JaCQUOT R., I96I $b$. Influence du niveau alimentaire sur le comportement nutritionnel de la Truie gestante. C.R. Acad. Sci. Paris, 253, I497-r 499.

Salmon-Legagneur E., 1963. Influence relative de l'azote et de l'énergie de la ration sur le comportement nutritionnel de la Truie gestante. Arch. Sci. Physiol., 17, 233-245.

Werner A., Hennig A., I960. Die biologische Wertigkeit des Futtereiweisses für Milchbildung und Erhaltung bei säugenden Sauen. Z. Tierphysiol. Tierern. Futtermitt., 15, I43-I 55 . 